Review Article

\title{
Damages of Underground Facilities in Coal Mines due to Gas Explosion Shock Waves: An Overview
}

\author{
Dezhi Ran, Jianwei Cheng ${ }^{D}$, Rui Zhang, Yu Wang, and Yuhang Wu \\ School of Safety Engineering, China University of Mining and Technology, Xuzhou 221116, China \\ Correspondence should be addressed to Jianwei Cheng; cheng.jianwei@cumt.edu.cn
}

Received 19 July 2021; Accepted 18 October 2021; Published 30 October 2021

Academic Editor: Pengfei Wang

Copyright (C) 2021 Dezhi Ran et al. This is an open access article distributed under the Creative Commons Attribution License, which permits unrestricted use, distribution, and reproduction in any medium, provided the original work is properly cited.

\begin{abstract}
With coal mining depth increase, gas explosion accidents due to the high gas emission rates often occur which cause significant casualties and property damages. Among them, gas explosion shock waves not only can destroy the machines and equipment in mine roadways but also cause the failure of mine ventilation facilities resulting in secondary hazards. Thus, the mines' serious disasters could happen. For many years, researchers have already done a great lot of works to study damages caused by the impact of shock waves of the gas explosions in underground mines. Research results provide a baseline for judgments of hazard effects by explosions. In this paper, the formation mechanism of the gas explosion shock wave is introduced firstly. Then, the damages for underground facilities, such as mechanical equipment, roadway, and life-saving devices are summarized and reviewed. Finally, a brief discussion about the methods is given, and some preliminary suggestions are also listed for improvements in the future.
\end{abstract}

\section{Introduction}

Coal is one of the important natural resources in the world. With the increase of mining depth, both the gas pressure and content in the coal seam gradually increase, which lead to a surge of gas emission rate and result in frequent gas explosion accidents $[1,2]$. In most cases, a gas explosion mainly causes high-temperature flames, highpressure shock waves, and amounts of toxic gases. The gas explosion instantly produces a large number of hightemperature flames and disaster gases; these flames and gases could quickly spread in the underground confined space, which can cause miners' casualties. Moreover, the generated explosion shock wave overpressure can damage the mine ventilation facilities causing mine airflow disorder [3]. The destruction of the mine ventilation system could cause the secondary explosion generated by the propagation of high-temperature flames in underground mine tunnels. The greatest impact caused by high-pressure shock waves not only destroys the roadway but also brings difficulties for workers performing rescues. Therefore, it is particularly important to study the impact and consequence of gas explosion damage.
The study of the main kinetic damage of gas explosion shock waves and the propagation law of explosion shock waves in the tunnel with different characteristics and the influence of gas explosion on the network structure of mine ventilation system not only have important theoretical and practical values but also are crucial to the prevention and prediction of gas explosion disaster accidents. Given this point, it is very necessary to review the current stage of research on gas explosion shock waves in mines.

\section{Gas Explosion Shock Waves}

The harmful factors generated by gas explosions are mainly flame fronts, shock waves, and toxic and hazardous gases [4]. The explosive mixture formed in coal mines is essentially a mixture of air and coal gas, consisting primarily of methane and small amounts of ethane and other alkanes. Although most accidental gas explosions in coal mines are deflagrations, the worst-case scenario includes explosions that can be extremely destructive, generating explosion pressures of up to $10 \mathrm{MPa}$ on reflection, which can destroy large amounts of equipment and facilities. And gas explosions can also cause secondary injuries, such as roof collapse accidents and 
bracket smashing accidents. After the impact of high-temperature and high-pressure gas, the relatively confined space of the coal mine, gas density reduction, and water evaporation could again form a low-pressure area; after the conditions are ripe, the surrounding gas can fill this area causing secondary explosions to occur, resulting in the severity of the accident increased. Gas explosion shock airflow (also known as burst wind) can aggravate the casualties and equipment damage. Shock airflow, immediately after the shock wave, is generated by the rapid expansion of explosive gas products, and the impact of the shock wave on people and obstacles generated by the pressure is called the shock wave dynamic pressure.

2.1. Explosion Pressure. Underground coal mine gas explosion can produce a hugely destructive effect because the explosion occurs in the confined space of the coal mine and can form a larger explosion pressure than the free space. Gas explosion pressure can usually be divided into two parts, which are the explosion overpressure and dynamic pressure. Theoretical and experimental evidence is the fact that air shock wave damage to the target depends not only on the shock wave overpressure but also on the overpressure of the action time. Therefore, scholars mainly used to take into account the value of the overpressure and the positive pressure area of the action time of the specific impulse to measure the shock wave method.

2.1.1. Explosion Overpressure. The shock wave is a sharp change in the state parameters of the sudden jump surface; the shock wave propagates in the tunnel when the wave front surface reaches a certain point; the air pressure rises instantaneously and far exceeds the atmospheric pressure; at this time, the air pressure above the atmospheric pressure part is called overpressure. After reaching the peak, due to the propagation of the explosion, the explosion products sweep more and more air mass, and the expansion rate slows down, equivalent to a series of expansion waves followed by the shock wave so that the shock wave surges up and the pressure continues to decay. Thus, the overpressure is reduced to zero and then appears below the surrounding gas pressure, which leads to the occurrence of a secondary recoil phenomenon. Eventually, it backs up to the normal atmospheric pressure. With the increasing gas concentration in the mine tunnel, the peak pressure of the gas secondary explosion rises and then falls, and the maximum value is slightly greater than the equivalent concentration [5].

2.1.2. Explosive Dynamic Pressure. Explosive dynamic pressure is the shock wave behind the front of high-speed movement of the air medium flow formation of impact pressure, with obvious directionality. The gas explosion was generated by many gas products in the role of high-temperature rapid expansion, and shock waves together with the synergistic movement and the explosion shock waves continue to strengthen and speed up, resulting in shock waves, and waves after the explosion products break off and break off the shock wave with the energy obtained from the explosion products that continue to propagate along the roadway. After the propagation of the shock wave, the pressure rises, and the gas behind the wave flows under the action of inertia, forming a shock airflow, which is known as the burst wind [6]. Although the duration of the shock airflow is not long, the speed is generally up to $100 \mathrm{~m} / \mathrm{s}$ or greater, and it can also make the formation of the impact on people and underground facilities and equipment to produce injury and damage.

2.2. Explosion Impulse. The specific impulse is directly determined by the overpressure versus time curve $P(t)$ of the air shock wave front and the positive pressure action time, where the magnitude of the positive specific impulse depends on the area enclosed by the positive pressure zone curve, and the magnitude of the negative specific impulse depends on the area enclosed by the negative pressure zone curve. The magnitude of the specific impulse can be obtained by the integral of the overpressure in time. We refer to the positive specific impulse by the specific impulse [7].

\section{Gas Explosion Shock Wave Attenuation}

Researchers' results are based on the gas explosion testing data conducted in the large size simulation tunnel. The decay model of the explosion shock wave overpressure is established. The relevant characterization parameters of the explosion shock wave are constantly corrected, researched to master the decay law of the shock wave overpressure in the experimental tunnel. Based on certain assumptions, the coupling equation of overpressure, air velocity, shock wave propagation velocity, and propagation distance after the decay of the strong shock wave of the gas explosion is established. It is found that the overpressure is inversely proportional to the propagation distance and roadway crosssectional area, and the overpressure is proportional to the total energy released by a gas explosion. The air velocity and shock wave propagation velocity are inversely proportional to the square root of propagation distance and roadway cross-sectional area, and both of them are all proportional to the square root of the total energy of the gas explosion [8].

3.1. Mathematical Model of Gas Explosion Propagation. For gas explosion as a rapid combustion reaction, both mathematical models and experimental simulations need to satisfy the following equations.

The continuous equation is expressed in the following equation:

$$
\frac{\partial \rho}{\partial t}+\frac{\partial(\rho u)}{\partial x}+\frac{\partial(\rho v)}{\partial y}+\frac{\partial(\rho w)}{\partial z}=0 .
$$

The energy equation is expressed in the following equation:

$$
\frac{\partial e}{\partial t}+u \frac{\partial e}{\partial x}+v \frac{\partial e}{\partial y}+w \frac{\partial e}{\partial z}=0
$$


The momentum equation is expressed in the form of the following equation:

$$
\begin{array}{r}
\frac{\partial u}{\partial t}+u \frac{\partial u}{\partial x}+v \frac{\partial u}{\partial y}+w \frac{\partial u}{\partial z}=-\frac{1}{p} \frac{\partial \rho}{\partial x} \\
\frac{\partial v}{\partial t}+u \frac{\partial v}{\partial x}+v \frac{\partial v}{\partial y}+w \frac{\partial v}{\partial z}=-\frac{1}{p} \frac{\partial \rho}{\partial y} \\
\frac{\partial w}{\partial t}+u \frac{\partial w}{\partial x}+v \frac{\partial w}{\partial y}+w \frac{\partial w}{\partial z}=-\frac{1}{p} \frac{\partial \rho}{\partial z}
\end{array}
$$

The equation of state is expressed in the form of the following equation:

$$
\begin{aligned}
P & =p(\rho, T) \\
& =\rho R T,
\end{aligned}
$$

where, in equations (1) (4), $p$ is the pressure, MPa; $t$ is the time, $s ; u, v$, and $w$ are the velocities in three coordinate directions, $\mathrm{m} / \mathrm{s} ; x, y$, and $z$ are right-angle coordinate system parameters, $m ; \rho$ is the fluid density, $\mathrm{kg} / \mathrm{m}^{3} ; T$ is the temperature, $\mathrm{K} ; R$ is the gas constant; $e$ is the specific energy, $e=p /(\gamma-1)+\rho\left(u^{2}+v^{2}+w^{2}\right) / 2$; and $\gamma$ is the gas index.

\subsection{Influencing Factors of Mine Gas Explosion Shock Wave.} Gas explosion shock waves include overpressure and dynamic pressure; they can show regular decay with the propagation of the explosion in the shaft. When the gas explosion is in a mine tunnel, the shock wave just propagates along the direction of the tunnel. Coal mines have relatively confined space; then, there is the same distance of infinite space explosion when the shock wave is much larger. The main factors affecting the peak overpressure of the air shock wave are the total energy of the gas explosion, the crosssectional area of the roadway, the initial state of the air, the roughness of the roadway, and the distance from the source of the explosion.

3.2.1. Total Energy of Gas Explosion $E_{0}$. The gas explosion energy comes from the heat of combustion reaction between gas and air; from the chemical equation of the reaction between $\mathrm{CH}_{4}$ and $\mathrm{O}_{2}, 1 \mathrm{~mol}$ of $\mathrm{CH}_{4}$ and $2 \mathrm{~mol}$ of $\mathrm{O}_{2}$ reaction give off the energy of $882.6 \mathrm{~kJ}$, according to the gas equation of state $\mathrm{PV}=\mathrm{nRT}$ to get the molar volume of gas at room temperature and pressure of $V=n R T / P=24 \mathrm{~L} \times \mathrm{mol}^{-1} .1 \mathrm{~m}^{3}$ of pure gas complete combustion (volume concentration of 9.5\%) released heat is $Q e=1000 / 24 \times 882.6=3.68 \times 104 \mathrm{~kJ}$. Then, for the concentration of $c$ and volume $V$ of the gas-air explosive gas mixture, its complete combustion when the heat is released can be calculated according to the following formula:

$$
E_{0}=Q_{e} \times V \times c, \mathrm{~kJ}
$$

3.2.2. Initial State Parameters of Air $p_{0}, \rho_{0}$. The shock wave is a strong compressional wave and is the result of the interaction and superposition of numerous weaker compressional waves. In the process of the shock wave along the roadway, the state parameters change abruptly, and the shock wave before and after the wave front surface of the state parameter changes has no continuity, accompanied by the generation of discontinuous between the new surface (shock wave front surface). To simplify the calculation, researchers often assume that the flow of gas explosion is a nonconstant flow (wave front surface state parameters change with time), and the atmospheric pressure of the underground air should be determined according to the actual situation in different coal mines.

3.2.3. The Cross-Sectional Area $S$ and Hydraulic Diameter $d_{0}$ of the Roadway. The larger the initial overpressure of the shock wave, the faster the shock wave attenuation, and the greater the rate of change in the cross-sectional area of the roadway, the greater the shock wave attenuation coefficient [9], where the hydraulic diameter can be derived from

$$
d_{0}=\sqrt{\frac{4 S}{\pi}}, m .
$$

3.2.4. Roughness of the Roadway $\beta$. The roughness of the roadway is an important factor in the decay of the peak shock wave overpressure, which varies under different support measures. The Soviet scholar M.A. Sadovsky et al. demonstrated that, for the same diameter of the roadway, the pressure drop due to friction on the air shock wave front in the range from 10 to $500 \mathrm{kPa}$ depended not only on the distance of the shock wave travels but also on the degree of roughness indicated by the roadway, and the equation for the pressure drop on the plane wave front taking into account the friction loss was given [10].

$$
\frac{\Delta P}{\Delta P^{\prime}}=e^{-\beta R / d_{0}},
$$

where $\Delta P$ is the overpressure of the air shock wave considering the friction of the roadway wall, $\mathrm{kPa} ; \Delta P^{\prime}$ is the overpressure of the air shock wave without considering the friction of the wall of the roadway, $\mathrm{kPa}$; and $\beta$ is the coefficient of the roughness of the roadway surface.

The roughness coefficient of the wall can also be calculated according to the corresponding formula in gas dynamics, and according to the previous research, it is found that when the overpressure value is $10 \mathrm{kPa} \leq \Delta P \leq 300 \mathrm{kPa}$, the error is small and can be used.

$$
\beta=\frac{2 g \alpha}{v}
$$

where $\alpha$ is the frictional resistance coefficient of the tunnel, constant when Reynolds $R e \geq 10^{5}$ (fluid is in a completely turbulent state). $v$ is the specific gravity of the air, $\mathrm{N} / \mathrm{m}^{3}$.

3.2.5. Distance from the Source of the Explosion L. For a certain amount of accumulation of explosive gas mixture generated by the shock wave overpressure value and the distance from the source has a direct relationship, the farther away from the source, the smaller the shock wave overpressure value. 
3.3. Effect of Ignition Energy on the Characteristics of Gas Explosion. With the extraction of gas for industrial and domestic use, there is a great knowledge gap in the safety production of the coal mining industry by a gas explosion in the pipeline. Qiu established the RNG $\kappa-\varepsilon$ turbulent flow field model based on the total energy equation and the step-bystep reaction explosion combustion model based on multiple control mechanisms, solved the explosion flow and reaction control equations by the finite volume method, and propagated the gas explosion under different ignition energy conditions. The conclusions showed that the greater the ignition energy, the greater the peak gas explosion pressure and flame propagation speed of the propagation law; the analysis of the positive feedback mechanism between the gas explosion pressure wave, explosion flame, and turbulence was an important factor to drive the development process of gas explosion [11].

\subsection{Effect of Obstacles on the Characteristics of Gas Explosion.} To study the propagation law of gas explosion in large size tunnels and the influence of obstacles on gas explosion, Jia established a two-dimensional model of mine tunnel using Fluent software to study the propagation law of gas explosion when the volume fraction of gas is $9.5 \%$. The results showed that the reaction of the explosion started from $25 \mathrm{~ms}$ and finished after $6 \mathrm{~ms}$, the flame propagation speed could reach $400 \mathrm{~m} / \mathrm{s}$, the maximum temperature could reach $3200 \mathrm{~K}$, the maximum temperature at the center of the tunnel reached $2155.5 \mathrm{~K}$, the maximum temperature of the tunnel was maintained at about $1,910 \mathrm{~K}$ after the reaction was completed, and the maximum value of the gas explosion shock wave was $1.08 \mathrm{MPa}$. When the obstacle existed, the shock wave at the obstacle shock wave rapidly increases. The closer the position of the obstacle to the explosion position, the greater the overpressure. The same position of different shapes of obstacles and round obstacle shock wave overpressure was the smallest [12]. Xu also found that when there was a square obstacle with a side length of $0.13 \mathrm{~m}$ in the pipe, the peak pressure during gas explosion reached $0.84 \mathrm{MPa}$; in the actual tunnel where there was a square obstacle, it especially should strengthen the arrangement of explosion isolation facilities; for different shapes of obstacles, with the increase of obstacle size, the peak explosion overpressure also increased [13]. Gao explored the effect of flexible obstacles in the flexible duct system and piping system of the auxiliary ventilation system on the gas explosion propagation law and established a gas explosion simulation model using the open-source CFD source generation OpenFOAM. The process variables in the XiFoam tool were used for deflagration, and the maximum pressure was increased by $29 \%$ and $77.8 \%$, respectively, and the maximum flame propagation speed was increased by $6.8 \%$ and $20 \%$, respectively, over the empty pipe flame propagation speed in the duct system and piping system models [14].

3.5. Effect of Different Section Shapes of Roadway on the Characteristics of Gas Explosion. Different section shapes have different stability degrees. Wu selected three different shapes of roadway sections according to the size and direction of ground stress at the site, used the same support method and construction method, and analyzed several sections for stability examples with parameters such as convergence displacement, displacement rate, and loosening range, thus obtaining that the influence of section shape on roadway support was huge, and the addition of bottom arch could not only prevent the bottom arch and prevent the bottom bulge but also suppress the displacement of the two gangs and the top [15]. Jia used circular, square, rectangular, and trapezoidal section test tubes to monitor the temperature and pressure changes to explore the propagation of gas explosions in the roadway. The results showed that the flame took the longest time to fill the alleyway with trapezoidal sections; however, the square section had the highest temperature. The maximum explosion pressure in the lane was about $0.6 \mathrm{MPa}$, but the lane with a trapezoidal section took the longest time period to reach the maximum explosion pressure, with the lowest gas explosion temperature [16].

3.6. Effect of Roadway Wall Conditions on the Characteristics of Gas Explosion. Ma estimated the effect of tunnel roadway conditions on gas-air explosions by numerical calculations and theoretical analysis. During the methane-air explosion, rough tunnel walls caused stronger turbulence. In the methane-air premixing zone, the effect of turbulence was manifested by a significant increase in explosion pressure. In the far zone of air shock wave propagation, turbulence reduced the shock wave intensity, but the effect was not significant. In the original methane-air premixing zone, the explosion pressure of the methane-air mixture in the roughwalled tunnel was higher than that in the smooth-walled tunnel. However, the air shock waves outside the premixed zone in the rough-walled tunnel were weaker than those in the smooth-walled tunnel [17].

\section{Impact of Explosion Shock Wave on Mine Ventilation Facilities}

4.1. Mine Fans. The fan is the heart of the mine, providing fresh air for the mine, excluding contaminated air, reducing the occurrence of gas explosions, coal dust explosions, and other disasters, and playing a pivotal role in the safe construction of the mine $[18,19]$. At present, many experts in related fields have conducted much research in the process of gas explosion shock wave propagation and already have a lot of theoretical knowledge; for example, Li et al. studied the explosion pressure and impact generated by large explosions through numerical simulation [20]; Ye et al. conducted theoretical and experimental research on the propagation law of flame and shock wave in the bend [21]; Song revealed the invented new dynamic response characteristics and laws of the explosion door of the vertical wind shaft during the explosion impact [22].

However, scholars have ignored the effect of explosion shock waves on the fan. Liu used Fluent simulation software to construct a three-dimensional model of the vertical shaftfan-explosion door to simulate the effect of different gas 
concentrations and explosion distances on the fan when the explosion door could not be opened normally [23]. The peak overpressure at the fan and explosion door increased with the increase of gas accumulation, and both showed a linear relationship. Analyzing the effect of different explosion distances on the fan and explosion door, the simulation results showed that the peak overpressure at the fan and explosion door decreased with the increase of explosion distance, both in a power function relationship, as shown in Figure 1.

4.2. Ventilation Network. The ventilation network is a topology consisting of hundreds of intricate tunnels and nodes; the main role is to convey the airflow, separating the new and dirty air, if the network chaos will lead to serious safety accidents [24]. The powerful pressure of the explosion shock wave exceeds the air supply pressure, which will cause the wind flow reversal and stagnation phenomenon, leading to the original network chaos, and thus make the scope of the disaster expand [25]. Underground gas explosion shock wave damage by the overpressure and positive pressure zone impulse jointly determine that when the shock wave propagation process will encounter obstacles, it will occur reflection, around the projection phenomenon.

$\mathrm{Li}$ and Cao constructed a three-dimensional network model of the mine ventilation system by studying the spreading law of smoke flow from underground fires and used visual implementation of a smoke flow spreading to simulate the dynamic spreading process of smoke flow in three-dimensional shafts, revealing the development of smoke temperature and concentration in space and time [26]. Yang studied dynamic characteristics of high-pressure shock airflow propagation in ventilation networks and the key nodes and flutter structure characteristics of ventilation networks and established the flutter structure characteristics analysis model of ventilation networks [27]. Qiu studied the propagation characteristics and attenuation law of explosion shock waves in flat, bend, bifurcation, A-type networks, and parallel networks and explored the overpressure at the bend or bifurcation of pipe attenuation coefficient and the bend or bifurcation angle and the initial pressure of the coupling relationship and explored the sudden change in its section overpressure attenuation coefficient, increase coefficient and reflection coefficient, respectively, with the section change rate, and the initial pressure of the coupling relationship [28]. At this stage, scholars mainly carry out the effect of the explosion on common types of networks given the actual situation that coal mine gas explosion may cause a large number of casualties, analyze the factors where gas explosion damages the ventilation system the most, and establish the gas explosion damage model.

An $\mathrm{H}$-shaped ventilation system means that the working face has 4 ventilation roadways ( 2 inlets and 2 return or 3 inlets and 1 return). The advantage of this ventilation system is that the working face has a large ventilation capacity and the gas does not gush to the working face. The disadvantage is that there is a large amount of roadway construction and maintenance. The maintenance of the roadway in the open area must prevent air leakage. Therefore, this type of ventilation system is also often used in the top coal comprehensive mining workings with a high gas release. With the improvement of coal mining mechanization, an $\mathrm{H}$-shaped ventilation system is mainly used at this stage, but then there is a great potential danger of gas explosion. Therefore, the $\mathrm{H}$-shaped ventilation system has become the object of research by many scholars. Ma used Fluent to simulate the process of gas explosion shock wave disrupting the normal ventilation of the tunnel to investigate the changes of pressure and airflow pattern during the process of gas explosion and venting in the $\mathrm{H}$-type tunnel; the molar fraction of $\mathrm{CO}_{2}$, temperature, and fluid movement in the tunnel before and after the explosion were compared and analyzed to obtain the propagation characteristics of the explosion shock wave in the tunnel. The results showed that the explosion shock wave was reflected by the wall of the tunnel and superimposed with the forward shock wave, increasing the pressure value in the superimposed area; the explosion shock wave changed the flow state of the airflow in the contact tunnel so that the airflow in the contact tunnel changed from double vortex mode to single vortex mode; the gas detonation process had a larger capacity of the tunnel on the side of the gas detonation area; and many shock waves generated by the explosion were discharged along the tunnel with a larger capacity. After the explosion, the tunnel could resume normal ventilation [29].

The common ventilation system is mainly $U$ type and $U+L$ type, coal mining working face mostly uses $U$ type ventilation, and high gas mine $U$ type ventilation working face of the upper corner gas has been a difficult problem of coal mine gas management. With the development of research, the high-production and high-efficiency mining process has been realized, the unit production of workings has been increasing, and the amount of gas gushing out has been increasing, so that mines with little gas in the original workings and low gas mines also have the upper corner gas accumulation over the limits. $U+L$ shaped ventilation system is evolved based on of $U$ shaped backward type. It adds a flat lane to the outer side of the working face or the backwind flat lane as a special gas discharge lane, commonly called "tail lane," forming a form of 1-in-2-back. The advantage of this ventilation system is that the air volume of the two backwind flat lanes can be controlled by adjusting the resistance, to control the amount of gas gushing to the working face from the mining area so that the upper corner does not exceed the limit. The disadvantage is that it increases the construction volume of 1 tailing lane and the maintenance volume of the lane is large. When the amount of gas gushing from the coal mining working face is very large, a special gas discharge lane can be used, but it must conform to the relevant provisions of the "Coal Mine Safety Regulations." At present, China's coal mining working face gas outflow is very large by the extraction of gas and increases; the amount of air still does not meet the requirements and often uses $U+L$ shaped ventilation system. For the above two ventilation systems on the destruction of a gas explosion, research is less. 


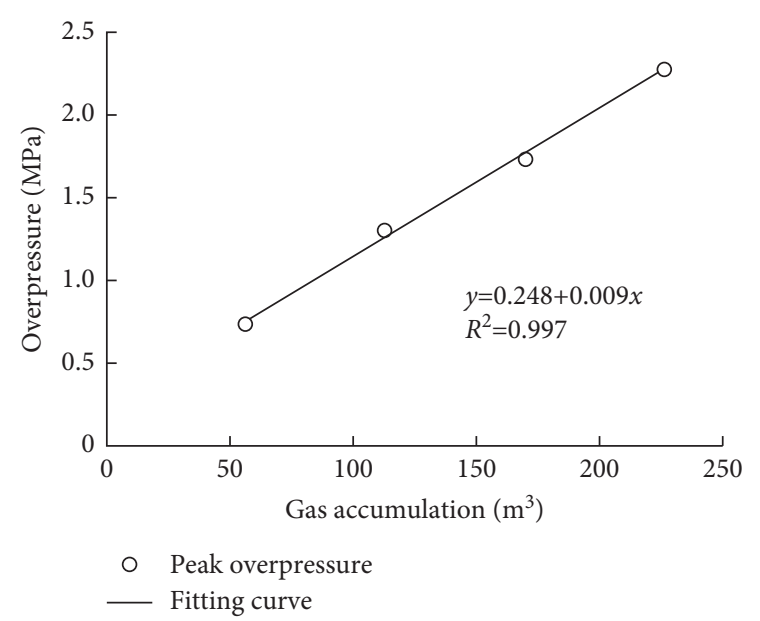

(a)

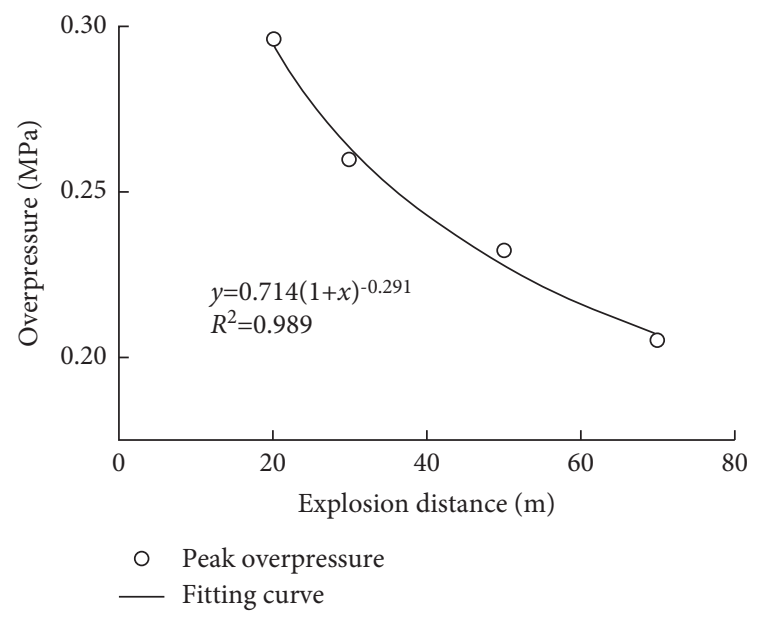

(c)

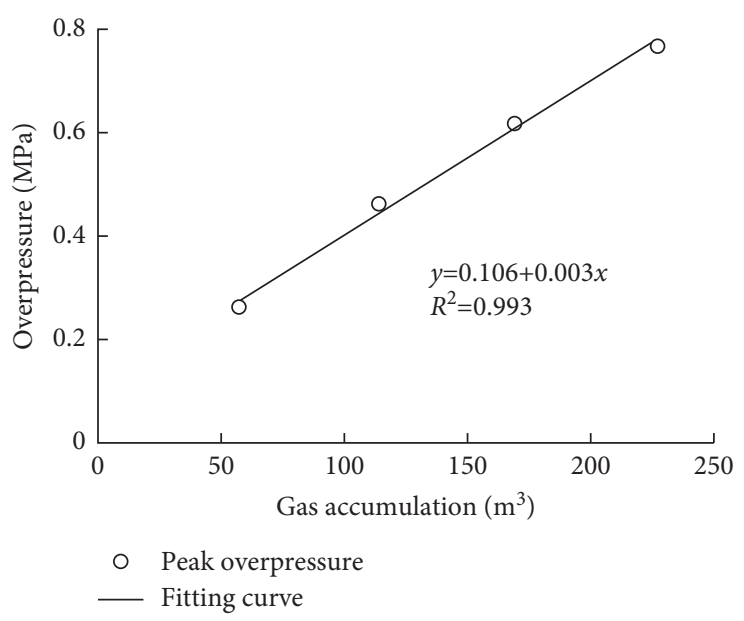

(b)

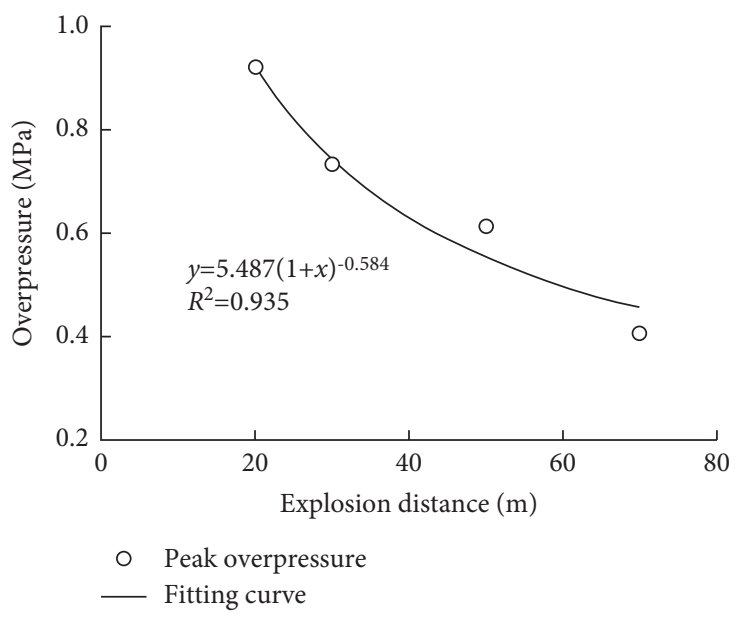

(d)

FiguRE 1: Fitting of influence law of overpressure peak value [23]. (a) Effect of gas accumulation on the peak overpressure at the explosion door. (b) Effect of gas accumulation on the peak overpressure at the fan. (c) Explosion distance on the peak overpressure at the fan. (d) Explosion distance on the peak overpressure at the explosion door.

4.3. Fireproof Mine Seal. Seals are used to control and direct ventilation airflow through underground coal mines, diluting and allowing harmless methane, carried coal dust, and other contaminants to enter the workings and other areas of the mine. They should be constructed using traditionally accepted methods and materials that have been proven to work adequately or methods and materials that have been tested and proven to have a minimum strength equal to or greater than traditionally accepted mine control.

A fundamental research direction at the National Institute for Occupational Safety and Health (NIOSH) was eliminating the occurrence or mitigating the effects of coal mine explosions. The researcher conducted a collaborative effort to develop and evaluate new innovative seal designs to assess the strength characteristics and resistance to air leakage of numerous innovative seal designs and ventilation control structures used in underground coal mines to provide enhanced explosion protection for mining personnel. To address the prevalent geologic mining conditions and loose and friable coal, encountered in establishing seals,
Eric S. Weis evaluated the effectiveness of using pressurized grout bags (packer bags) along the mine roof and veins instead of bottom and vein couplings for standard types of solid concrete block seals. The results showed that the use of these fast-setting grout-filled packer bags, internally pressurized to $300 \mathrm{kPa}$, not only provided a seal that could withstand a $138 \mathrm{kPa}$ static pressure explosion but also provided a better seal than the joint ribs.

Zhang used numerical simulation to investigate the mechanical properties of brick seals under impact loading [30]. The characteristics of gas explosion in the confined area and the propagation characteristics of the shock wave were analyzed, and the pressure variation of the shock wave on the seal caused by the gas explosion was predicted. A series of numerical simulations of the brick seal under different pressure variations were carried out to obtain the safety variations of the brick seal under different conditions, as shown in Figure 2.

The analysis of the explosion process showed that when the gas explosion occurred, the maximum shock pressure of 


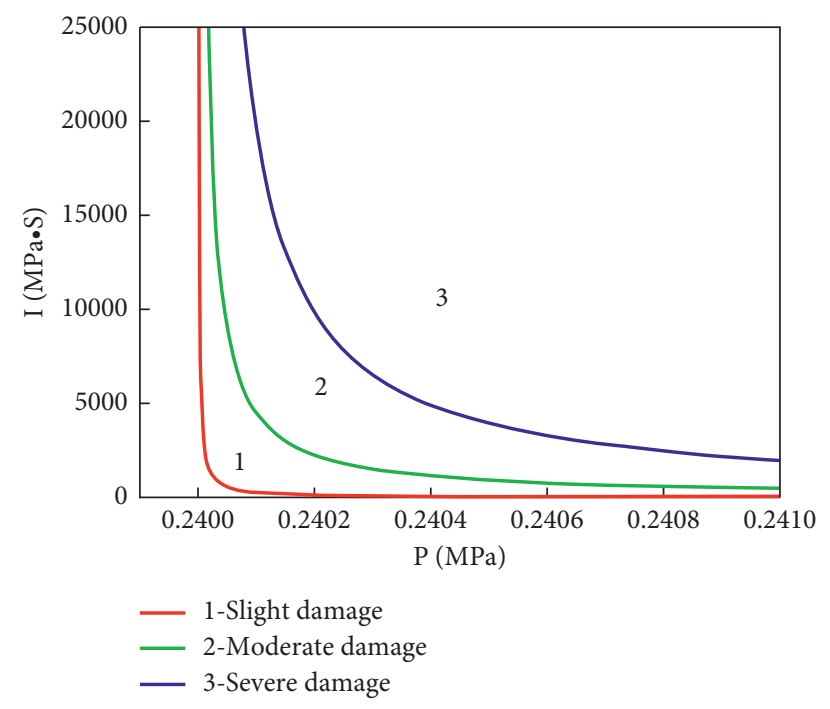

Figure 2: Damage curve of $1.5 \mathrm{~m}$ thick brick seal [30].

the quasi-static structure reached $0.83 \mathrm{MPa}$; when the dust explosion occurred, the possible explosion pressure was between 0.79 and $0.89 \mathrm{MPa}$. However, due to the different spatial structure, when there was sufficient propagation distance, there would be a dynamic increase in the quasistatic basis; when the explosion pressure might rise to 1.75 $\mathrm{MPa}$, this shock wave would be reflected when propagating to the seal; the shock wave and the reflected wave superimposed on the impact pressure were larger, $4.50 \mathrm{MPa}$, and the use of the impact load under the action of the brick seal damage variable $D$, respectively, was defined as $1 / 5$ (minor damage of brick seal layer), 1/3 (moderate damage), and $1 / 2$ (serious damage) state judgment method; by analyzing the degree of damage of brick seal layer under the action of different impact loads, fitting the curve, the damage curve of single brick seal layer was derived.

\section{Impact of Explosion Shock Wave on Mechanical Equipment}

Mine machinery and equipment mainly include brackets, coal mining machines, and transporters, where the role of the bracket is to support the stability of the overlying rock layer. However, scholars at home and abroad have less research on the damage to mechanical equipment caused by explosion shock waves; if the shock waves damage the bracket, they not only block the passage of people in the tunnel but also easily cause smashing accidents caused by the collapse of the roof. We can use the form of accident tree analysis and give a certain weight to mechanical equipment and a comprehensive assessment of the risk of explosive accidents, only through a variety of preventive measures to achieve a significant reduction in the risk of explosion. The use of numerical and experimental simulations can only be a general design of the appearance of mechanical equipment, ignoring the details. Although most of the mechanical equipment is intrinsically safe or explosion-proof, at this stage, there are still great problems, especially with electrically charged equipment; shock wave destruction of equipment may also lead to the explosion-proof performance of electrical equipment failure, resulting in greater accidents, so shock wave damages and explosion-proof performance of electromechanical equipment failure chain research have great prospects for development.

\section{Impact of Explosion Shock Wave on the Underground Moveable Rescue Chamber}

The life-saving cabin is a kind of underground rescue equipment, as shown in Figure 3. The life-saving cabin can isolate toxic and harmful gases in case of the mine disaster, provide necessary conditions for human survival such as oxygen and food, and prolong the survival time of riskaverse personnel; in the event of an explosion accident, we mainly need to resist the huge gas shock wave pressure and need to have strong antidetonation performance, but there are still great problems about the rescue chamber mechanism design.

6.1. Overall Research. In response to the current problem of the quantitative relationship between the factors affecting the explosion resistance of the rescue chamber and the increase in mass, Wang studied the improvement of the explosion resistance of different sections of the rescue chamber under the factors of structural form change, outer skin thickening, reinforcement thickening, and flange thickening using finite element calculation method based on an actual type of cabin, calculated the value of the increase in mass of the cabin under different factors, and analyzed the explosion resistance. The quantitative relationship between the increase of explosion strength and weight was analyzed. Research findings showed that when increasing the same quality, the thickness of the reinforcement on the cabin explosion resistance of the most obvious effect is more than the skin thickening which is 5.3 times higher than the flange thickening which is 2.7 times higher. Thickening the 


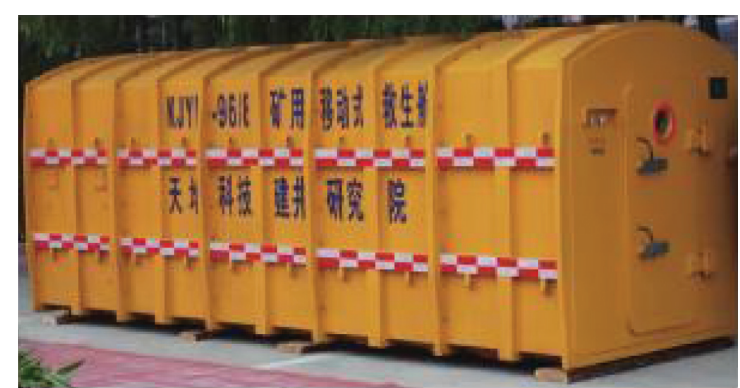

FIgURE 3: Moveable rescue chamber [31].

reinforcement was to enhance the cabin explosion resistance of the most economical and reasonable method. The design of the rescue chamber not only requires compliance with the current national standards but also requires convenience and high safety, as shown in Table 1 [31].

The coal mine rescue chamber becomes important equipment for miners to escape from underground, and its structural strength directly determines the life safety of miners. To improve the strength of the rescue chamber, Yang established a finite element model of the rescue chamber in the ABAQUS environment and used the explicit integration method to obtain the effect of the pressure on the rescue chamber during the coal mine gas explosion. The maximum stresses in the rescue chamber were found in the corner stress concentrations and the middle part of the ribs and reached the yield strength, but the overall stresses in the rescue chamber were within the allowable range [32].

Bai used the explicit kinetic analysis software LS-DYNA to study the TNT equivalent of gas explosion in the underground tunnel of the rescue chamber, the propagation characteristics of the shock wave, and the impact load on the cabin, and the propagation process of the explosion shock wave was obtained by simulation with the ALE fluid-solid coupling algorithm. When the shock wave was transmitted from the front end to the rear end of the rescue chamber and decays to $10 \%$ of its peak load, the TNT equivalent of gas when the load on the cabin reached the specification was determined, and the actual load curve of each part of the cabin was obtained, which provided a basis for calculating the dynamic response of the rescue chamber to impact when the load was applied and for optimizing the design [33].

Jing used the finite element software AUTODYN, using the principle of fluid-solid coupling, to simulate the explosion of rectangular section, circular section, and an arch section rescue chamber, respectively, to obtain the surface impact load distribution of these three different sections of the rescue chamber in the explosion flow field. It could be concluded that, under the same conditions, the surface impact load of the arch-shaped rescue chamber was the smallest among these three types of rescue chambers [34].

6.2. Refuge Door Research. In the event of an accident, emergency underground refuge chambers play an important role in ensuring the safety of trapped personnel. In the structural dynamic response of a refuge door under explosion, the load was investigated in a finite element model based on the FEM platform. Before entering the physical prototype pit explosion test, it provides a theoretical basis and data reference for further performance improvement of the product [35].

In the structural dynamic response of a coal mine refuge door under explosion, the load was investigated by building a finite element model based on the FEM platform. The results show, that under the action of $0.6 \mathrm{MPa}$ shock wave, the stress and deformation of the protective door meet the requirements, and the airtightness was good, which provides a theoretical basis and data reference for further improvement of the performance of the physical prototype before entering the explosion test in the pit [36], as shown in Figure 4.

The main design parameters of the explosion-resistant structure of the refuge door were determined by theoretical calculations, and the ANSYS/LS-DYNA software was used to perform dynamic finite element numerical simulations of the explosion-resistant structure of the refuge door. The results of the simulations and the explosion tunnel tests showed that the explosion-resistant structure of the protective door could resist the explosion impact pressure of not less than $0.5 \mathrm{MPa}$ and ensure its safety and reliability in the underground refuge protection [37], as shown in Figure 5.

Under the action of the triangular shock wave pressure of $0.5 \mathrm{MPa}$, the maximum displacement of the overall structure appears at $t=0.111 \mathrm{~s}$, and the maximum value of the displacement of the overall structure appears in the middle position of the door body, reaching $0.528 \mathrm{~mm}$, the displacement value gradually decreases from the middle to all around, and the displacement value of the overall structure is within $0-0.528 \mathrm{~mm}$.

\section{Impact of Explosion Shock Wave on the Roadway}

Coal mine gas explosion is extremely dangerous and can cause mass death and injury in a relatively short time. And once the accident occurs, the explosion wave will also damage the roadway surrounding rock materials and structure, so that the roadway failure damages, and even collapses, resulting in trapped personnel, and then directly impedes the rescue work that should be carried out, bringing great difficulties to rescue [38].

$\mathrm{Lu}$ found that the deep coal rock body under the coupling effect of gas explosion load and high stress and the stress time curve in the surrounding rock was characterized by multiple wave peaks, and the tunnel surrounding rock was successively subjected to shock waves, compression waves, and elastic waves, corresponding to the generation of multiple stress wave peaks, negative pressure zones, and obvious stress oscillations. In the corner of the roadway stress curve, there was a certain lag period, and with the increase of ground stress, the lag period would be reduced or even disappear. Under the coupling effect of explosion load and initial stress, stress concentration would appear at the corner of the tunnel top and bottom plates, showing obvious expansion and deformation in both horizontal and vertical directions, resulting in macroscopic damage at the corner of 
TABLE 1: Mass and explosion resistance increase table under different factors [31].

\begin{tabular}{lcccc}
\hline No. & Factors (\%) & $\begin{array}{c}\text { Mass increase } \\
(\%)\end{array}$ & $\begin{array}{c}\text { Increase in explosion resistance } \\
(\%)\end{array}$ & $\begin{array}{c}\text { The ratio of increase in } \\
\text { explosion } \\
\text { resistance to increase } \\
\text { in mass }\end{array}$ \\
\hline 1 & Skin thickness increased 50 & $18.6-18.8$ & $9.0-17.7$ & $0.48-0.94$ \\
2 & Stiffener increased 100 & $3.6-4.6$ & $10.2-21.7$ & $2.83-4.72$ \\
3 & Flange thickness increased & $26.5-26.8$ & $34.0-39.8$ & $1.28-1.48$ \\
\hline
\end{tabular}

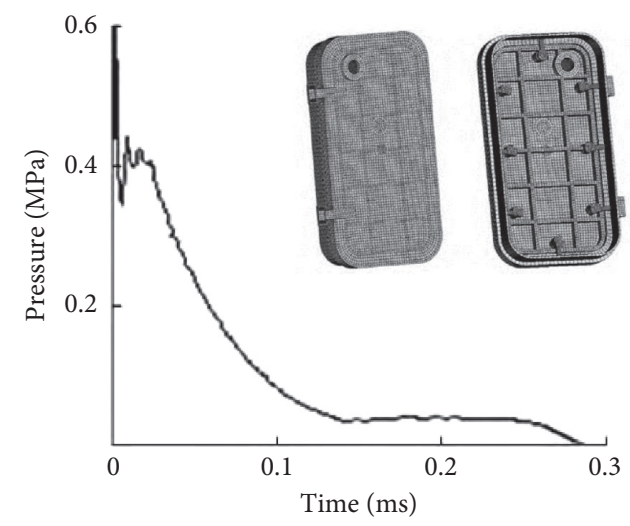

FIGURE 4: Shock wave pressure curve of the protective closed door [36].

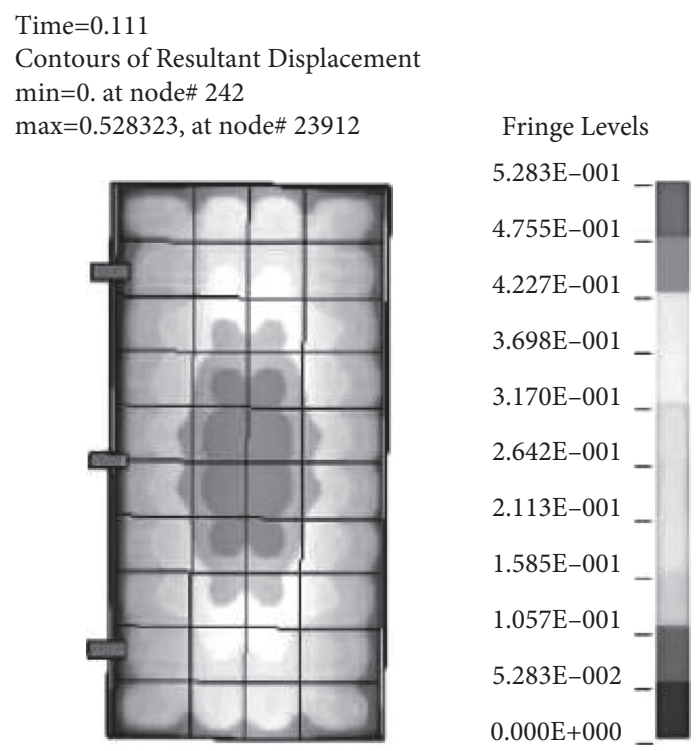

FIGURE 5: Maximum displacement [37].

the tunnel, but no obvious macroscopic damage at the two walls and roof and floor. The transient unloading caused by gas explosion mainly occurs in a small part of the area around the two gangs and the top and bottom of the roadway, and with the increase of the lateral pressure coefficient, the range of plastic damage to the surrounding rock of the two gangs of the roadway decreases [39].

Wang used numerical analysis to study the explosion resistance of underground chambers under different directional explosion loads. Through comparative analysis of the time course of the explosion compressive stress curve and dynamic crack distribution pattern of the underground chamber and the anchored chamber under the same equivalent explosion load, the top explosion produced the smallest displacement, followed by the arch side explosion, the largest in the straight wall side explosion, and the straight wall side explosion produced the largest displacement position which is the bottom of the sidewall, rather than the location of the closest explosion source cavity wall. During the explosion load, due to the reinforcement of the anchor 
rods, tensile "split" occurs between the anchor rods, and at the same time, the phenomenon of "spalling" occurs in the anchoring area and the end of the anchoring area. Under the same equivalent top explosion, arch side explosion, and straight wall side explosion, the displacement and dynamic crack distribution of the anchored chamber are similar, and the explosion resistance of the anchor reinforced chamber is improved [40].

\section{Safety Distance Study to Avoid Explosion Shock Wave}

The safety distance in a general term refers to the minimum straight-line distance from the center of the explosion to the operator not being injured by the explosion wave in the event of a gas explosion underground [41]. Safety distance is a relative concept and can be understood from both safety and injury-causing perspectives. In the former case, the personnel can be protected from the explosion shock wave outside the distance; in the latter case, the personnel within the distance will be injured.

After the underground gas explosion, the explosion shock wave and shock airflow will be propagated along the underground roadway; under the influence of friction and other heat losses in the wall of the roadway, its intensity decays. In the process of explosion propagation, to prevent the explosion shock wave on the underground operators that caused injury and ensure safety, we must determine the human body and underground ventilation structures to cause certain destruction of the critical shock wave parameters and to ensure that the operator is located in the location of the shock wave parameters less than the critical value, to ensure a certain safety factor. Polish scholars based on a statistical analysis of gas explosion accidents stated that mine gas explosion $500 \mathrm{~m}$ away from the source has two $90^{\circ}$ bend locations, which can be used as a safe distance for rescue and relief personnel [42].

\section{Discussion}

At this stage, scholars mainly use numerical simulation, but the gas explosion is in various forms, and the pattern is still complex. The energy release coming from an explosion is huge. The energy source location is random, considering the influence of many aspects, so the model construction, initial conditions, boundary conditions set, and monitoring point location selection are very problematic. The possibility of gas explosion in the underground mining face is large, but the mathematical model is too large to solve; to solve the mine full-scale gas explosion is difficult to simulate the problem; the main summer at this stage uses the explosion shock wave propagation segmental relay simulation method; the return wind alley pressure is the initial pressure of the winding shaft that converted to the bottom of the shaft gas explosion that occurred in the explosion equivalent.

In the form of experiments, simulating the mine environment in proportion to its complexity and pipe wall reduction is still only in the theoretical stage, there is a large deviation, and the study of the impact of gas explosion is simply considered a single variable and the experimental design to strive for simplicity, which led to the study of gas explosion mechanism stagnation. A gas explosion is a huge energy, for the relevant control and rescue devices to put forward a great demand for explosion resistance. On the research of pressure-resistant materials and device design, there is a good prospect. The gas explosion caused by the danger not only requires the use of safety system engineering means to consider multiple factors, but the most important thing is also to achieve a real strengthening of personnel management.

\section{Conclusions}

This paper summarizes the latest research results of gas explosion shock wave damage to ventilation facilities, mechanical devices, roadways, and rescue chambers, with the primary goal of pointing the way to future research and design for explosion resistance in coal mines. Rather than considering the explosion resistance of a single mine facility, this overview takes a systematic approach to the overall mine facility, including not only experimental and numerical simulation findings but also an overview of the use of new technologies.

In short, there is still a lot of room for development for gas explosion damage research, at this stage, mainly using a combination of experimental and simulation methods, of which numerical simulation is the development trend, but for the boundary conditions and the initial conditions, we still need a lot of experimental data. Regarding gas extraction and its complex construction process, there may be many safety risks of the gas explosion, but there is less research on the impact of gas shock waves on the gas extraction damage of gas extraction devices.

\section{Data Availability}

The data used to support the findings of this study are included within the article.

\section{Conflicts of Interest}

The authors declare that there are no conflicts of interest regarding the publication of this paper.

\section{References}

[1] L. Qi, "Analysis of the hazards of coal mine gas and integrated control measures," Energy and Energy Conservation, vol. 4, pp. 126-128, 2013.

[2] W. Song, J. Cheng, W. Wang et al., "Underground mine gas explosion accidents and prevention techniques- an overview," Archives of Mining Sciences, vol. 66, no. 2, pp. 297-312, 2021.

[3] H. Yu and J. G. Wu, "Study on control technology for mine gas explosion," Coal Technology, vol. 29, no. 10, pp. 85-87, 2010.

[4] I. Ivanov, A. M. Baranov, S. Akbari, S. Mironov, and E. Karpova, "Methodology for estimating potential explosion hazard of hydrocarbon with hydrogen mixtures without identifying gas composition," Sensors and Actuators B: Chemical, vol. 293, 2019. 
[5] L. Wen, "Research on secondary explosion process and its law in confined space," Master thesis, Liaoning University of Engineering and Technology, Liaoning, China, 2018.

[6] S. Yang, G. Jing, and Z. Jia, "Injury study on impact current of gas explosion in coal mine," Journal of China Coal Society, vol. 34, no. 10, pp. 1354-1358, 2009.

[7] W. Zhou, "Numerical analysis of explosive blast shock wave spread and dynamic instability of spherical shell," Master thesis, Taiyuan University of Technology, Taiyuan, China, 2010.

[8] B. Jiang, "Multiparameter temporal and spatial evolution of gas explosion and its suppression using gas-olid jet curtain," PhD thesis, China University of Mining and Technology, Beijing, China, 2014.

[9] K. Wang, A. Zhou, W. Gaoju, and Z. Pin, "Influences of roadway cross-section variation on outburst shock wave propagation," Journal of China Coal Society, vol. 37, no. 6, pp. 989-993, 2012.

[10] H. Tan, "Discussion on the effect of air blasting wave of blasting operation on the stability of the tunnel's country rock," Gold, vol. 4, pp. 24-25, 1998.

[11] R. Qiu, "Numerical simulation study of gas explosion dissemination in ingnition energy," Safety In Coal Mines, vol. 42, no. 1, pp. 5-8, 2011.

[12] J. Jia and J. Su, "Study on the influence of obstacles on gas explosion in driving face," Shanxi Science Technology, vol. 35, no. 6, pp. 21-23+27, 2020.

[13] A.-M. Xu, X. X. Chen, and J. Jia, "Effects of obstacles on gas explosion shock wave propagation," China Safety Science Journal, vol. 29, no. 9, pp. 96-101, 2019.

[14] G. Ke, S. Li, Y. Liu, J. Jia, and X. Wang, "Effect of flexible obstacles on gas explosion characteristic in underground coal mine," Process Safety and Environmental Protection, vol. 149, 2021.

[15] Z. Wu, Y. Luo, and B. Qin, "Study on the influence of section shape on the stability of roadway," Mine pressure and roof management, vol. 4, pp. 41-43+40, 2004.

[16] J. Jia and F. Wang, "Propagation of gas explosions in roadways with different cross-sectional shapes," Energy Sources, Part A: Recovery, Utilization, and Environmental Effects, vol. 43, p. 4, 2021.

[17] Q. J. Ma, Q. Zhang, and L. Pang, "Influence of the tunnel wall surface condition on the methane-air explosion," Combustion, Explosion and Shock Waves, vol. 50, p. 2, 2014.

[18] J. Cheng, X. Zheng, W. Luo et al., "A compound binder of coal dust wetting and suppression for coal pile," Process Safety and Environmental Protection, vol. 147, pp. 92-10, 2020.

[19] J. Cheng, Y. Wang, Y. Lei et al., "Study on coal dust crusting for coal pile based on a compound binder," Powder Technology, vol. 376, pp. 149-166, 2020.

[20] J. Li and H. Hong, "Numerical and analytical prediction of pressure and impulse from vented gas explosion in large cylindrical tanks," Process Safety and Environmental Protection, vol. 127, 2019.

[21] Y. Qing, L. Bai-quan, Z.-z. Jia, and C.-j. Zhu, "Propagation law and analysis of gas explosion in bend duct," Procedia Earth and Planetary Science, vol. 1, p. 1, 2009.

[22] W. Song, "Study on the safety protection theory and technology of explosion doors for vertical shafts in coal mines," $\mathrm{PhD}$ thesis, Henan University of Technology, Zhengzhou, China, 2018.

[23] J. Liu, S. Chen, J. Ren, and J. Hu, "Research on influence of gas accumulation amount and explosion distance on fan and explosion-proof door," Journal of Safety Science and Technology16, vol. 9, pp. 57-63, 2020.

[24] J. Cheng, W. Luo, Z. Zhao et al., "Controlling coal spontaneous combustion fire in longwall gob using comprehensive methods-a case study," Mining, Metallurgy \& Exploration, vol. 38, no. 4, pp. 1801-1816, 2021.

[25] R. Zhang, J. Cheng, Z. Wang, and Z. Shao, "Recapitulation and prospect of research on flow field in coal mine gob," Shock and Vibration, vol. 2021, Article ID 3730606, 24 pages, 2021.

[26] C. Li, Z.-G. Cao, Z.-X. Li, L. Hu, and D. Hou, "3D simulation modeling techniques of fire fume spread process for underground mines," Journal of China Coal Society, vol. 38, no. 2, pp. 257-263, 2013.

[27] Y. Yang, "Study on the dynamics effect of gas explosion in the mine ventlitation network," PhD thesis, Anhui University of Technology, Ma'anshan, China, 2011.

[28] J. Qiu, "Study on propagation characteristics of shock wave in pipe network and simulation of catastrophic process for gas explosion," $\mathrm{PhD}$ thesis, Anhui University of Technology, Ma'anshan, China, 2018.

[29] H. Ma, X. Chen, and D. Jing, "Simulation study on gas explosion and discharge process in H-type ventilation roadway," China Safety Science Journal, vol. 31, no. 1, pp. 45-51, 2021.

[30] X. Zhang, J. Cheng, and C. Shi, "Damage assessment for underground brick seal under explosion impact load," Arabian Journal of Geosciences, vol. 14, p. 5, 2021.

[31] L. Wang, "Relationship between factors affecting anti-explosion strength and weight increase of mine refuge chamber," Safety In Coal Mines, vol. 51, no. 12, pp. 163-166, 2020.

[32] X. Yang, L. Jin, and J. Li, "Explosion simulation and analysis of coal mine rescue capsule," Coal Mine Machinery, vol. 33, no. 2, pp. 113-114, 2012.

[33] B. Bai, Z. Li, Z. Wang, and L. Zhao, "Research on the tnt equivalent and impact load in anti-explosion of mine rescue capsule," Science Technology and Engineering, vol. 14, no. 17, pp. 1-5+20, 2014.

[34] J. Cheng, F. Liu, Y. Shi et al., "Model tests of effects on fire smoke control in highway tunnels," Journal Gradevinar (Civil Engineer), vol. 72, no. 9, pp. 781-792, 2020.

[35] Z. Jing, A. Zhang, B. Zhang, and Y. Gu, "Simulation analysis of explosion impact load on different cross-section rescue capsule," Coal Mine Machinery, vol. 34, no. 5, pp. 103-105, 2013.

[36] W. Wang, Y. Ni, L. Dan, and W. Lei, "Structural dynamic analysis of coal mine refuge chamber protective closure doors based on finite element method," Hoisting and Conveying Machinery, vol. 06, pp. 45-48, 2017.

[37] Z. Liu, "Dynamic analysis on finite element structure of protective sealing door applied to underground mine refuge chamber," Mine Construction Technology, vol. 37, no. 3, pp. 41-43, 2016.

[38] B. Zhige, "Research on anti-explosion performance of mine refuge chamber airtight blast door," Mining Safety \& Environmental Protection, vol. 39, no. 6, pp. 18-22+101, 2012.

[39] X. Lu, "Research on roadway damage mechanism under coupling of gas explosion load and the high ground stress," Master thesis, China University of Mining and Technology, Beijing, China, 2019.

[40] G. Wang, C. Wang, Y. Yu, and X. Chang, "Research on anchored effect of tunnels under explosion load in different directions," Chinese Journal of Underground Space and Engineering, vol. 13, no. 6, pp. 1645-1653, 2017.

[41] X. Lang, "Study on the aettenuation law and safe distance of gas explosion shock wave," Master thesis, China University of Mining and Technology, Beijing, China, 2015.

[42] S. Wang and Z. Xie, "Exploration on the safety distance of methane explosion in mines," Journal of China University of Mining \& Teehnology, vol. 4, pp. 4-11, 1989. 